\title{
Note on Bicomplex Matrices
}

\author{
Canan Ölçek and Semra Kaya Nurkan*
}

(Communicated by Bülent ALTUNKAYA)

\begin{abstract}
In this paper, we consider bicomplex numbers and bicomplex matrices. Firstly, we give some properties of bicomplex numbers. After that we investigate bicomplex matrices using properties of complex matrices.Then we define the complex adjoint matrix of bicomplex matrices and we describe some of their properties. Furthermore, we give the definition of q-determinant of bicomplex matrices.
\end{abstract}

Keywords: Complex number; Bicomplex number; Complex matrix; Bicomplex matrix.

AMS Subject Classification (2010): 15B33; 11E88; 11 R52.

${ }^{*}$ Corresponding author

\section{Introduction}

The subject of multiple imaginary units was examined in the 1840s. In a long series "On quaternions; or on a new system of imaginaries in algebra" beginning in 1844 in Philosophical Magazine, William Rowan Hamilton communicated a system multiplying according to the quaternion group. In 1848 Thomas Kirkman reported on his correspondence with Arthur Cayley regarding equations on the units determining a system of hypercomplex numbers.

It is possible to arrive to bicomplex numbers by means of purely algebraic considerations. For example, if in a complex number $a+i b$ we replace the real numbers $a$ and $b$ by complex numbers $z_{1}=a_{1}+i a_{2}$ and $z_{2}=b_{1}+i b_{2}$, then we get just another complex number:

$$
z_{1}+i z_{2}=\left(a_{1}+i a_{2}\right)+i\left(b_{1}+i b_{2}\right)=\left(a_{1}-b_{2}\right)+i\left(a_{2}+b_{1}\right)
$$

If we want to obtain a new type of number, then we must use another imaginary unit, say $j$, with $j^{2}=-1$, and set

$$
z_{1}+j z_{2}=\left(a_{1}+i a_{2}\right)+j\left(b_{1}+i b_{2}\right)
$$

which gives a new object, outside the field of complex numbers.

If we want to be able to operate with these new numbers, we need to define the product of the two imaginary units. This was a problem that was solved by Hamilton by requiring that they anticommute, and his solution led to the introduction of quaternions. Hamilton's decision was influenced by many considerations, including the desire to obtain a field, which of course the quaternions form (a skew field). But one could explore what happens if we assume that the two new imaginary units commute. In this case we obtain a new and lesser known object, the algebra of bicomplex numbers.

The set $B \mathbb{C}$ of bicomplex numbers is therefore defined as follows:

$$
B \mathbb{C}=\left\{z_{1}+j z_{2} \mid z_{1}, z_{2} \in \mathbb{C}\right\},
$$

where $i$ and $j$ are commuting imaginary units i.e $i j=j i, i^{2}=j^{2}=-1$ and which $\mathbb{C}$ is the set of complex numbers with the imaginary unit $i$. Thus bicomplex numbers are "complex numbers with complex coefficients", which

Received : 14-11-2017, Accepted : 03-10-2018 
explains the name of bicomplex, and in what follows we will try to emphazise the deep similarities between the properties of complex and bicomplex numbers. We should probably point out that bicomplex numbers were apparently first introduced in 1892 by Corrado Segre, that the origin of their function theory is due to the Italian school of Scorza-Dragoni and that a first theory of differentiability in $B \mathbb{C}$ was developed by Price.

Now the addition and the multiplication of bicomplex numbers are introduced in a natural way: given $Z_{1}=z_{1}+j z_{2}$ and $Z_{2}=z_{3}+j z_{4}$ in $B \mathbb{C}$, then

$$
Z_{1}+Z_{2}:=\left(z_{1}+z_{3}\right)+j\left(z_{2}+z_{4}\right)
$$

and

$$
Z_{1} \cdot Z_{2}:=\left(z_{1}+j z_{2}\right)\left(z_{3}+j z_{4}\right)=\left(z_{1} z_{3}-z_{2} z_{4}\right)+j\left(z_{1} z_{4}+z_{2} z_{3}\right) .
$$

So $(B \mathbb{C},+,$.$) is a commutative ring, i.e.$

1) The addition is associative, commutative, with identity element $0=0+j 0$, and all bicomplex numbers have an additive inverse. This is to say that $(B \mathbb{C},+)$ is an Abelian group.

2) The multiplication is associative, commutative, with identity element $1=1+j 0$.

3) The multiplication is distributive with respect to the addition i.e. for any $Z, Z_{1}, Z_{2} \in B \mathbb{C}$, we have:

$$
Z\left(Z_{1}+Z_{2}\right)=Z Z_{1}+Z Z_{2}
$$

The complex conjugation plays an extremely important role for both algebraic and geometric properties of $\mathbb{C}$, and for analysis of complex functions. It appears that there are three conjugations in $B \mathbb{C}$ which is not surprising: indeed, the complex conjugation is totally given by its action over the imaginary unit; thus one expects at least two conjugations on $B \mathbb{C}$ but one more candidate could arise from composing them. Consider these ideas in more detail.

Bicomplex conjuations with respect to $i, j, i j$ are determined respectively by the formulas

$$
\begin{aligned}
\bar{Z}(i) & :=\overline{z_{1}+j z_{2}}=\overline{z_{1}}+j \overline{z_{2}} \\
\bar{Z}(j) & :=\overline{z_{1}+j z_{2}}=z_{1}-j z_{2} \\
\bar{Z}(i j) & : \quad=\overline{z_{1}+j z_{2}}=\overline{z_{1}}-j \overline{z_{2}} .
\end{aligned}
$$

\section{Bicomplex Matrices and Their Adjoints}

The set $M_{m \times n}(B \mathbb{C})$ denotes all $m \times n$ type matrices with bicomplex numbers entries.If $m=n$, then the set of bicomplex matrices is denoted by $M_{n}(B \mathbb{C})$. The ordinary matrix addition and multiplication are defined. With these operations $M_{n}(B \mathbb{C})$ is a ring with a unit. The left (right) scalar multiplication is defined as, for $A=\left(a_{s t}\right) \in$ $M_{m \times n}(B \mathbb{C}), Z \in B \mathbb{C}$

$$
\begin{aligned}
& Z A=\left(Z a_{s t}\right) \\
& A Z=\left(a_{s t} Z\right) .
\end{aligned}
$$

But it is easy to see that

$$
Z A=A Z
$$

for bicomplex matrices because the product of bicomplex numbers is commutative.

For $A=\left(a_{s t}\right) \in M_{m \times n}(B \mathbb{C}), \bar{A}=\left(\overline{a_{s t}}\right) \in M_{m \times n}(B \mathbb{C})$ is the conjugate of $A ; A^{T}=\left(a_{t s}\right) \in M_{n \times m}(B \mathbb{C})$ is the transpose of $A ; A^{*}=(\bar{A})^{T} \in M_{n \times m}(B \mathbb{C})$ is the conjugate transpose of $A$.For a square matrix $A \in M_{n}(B \mathbb{C})$ if $A A^{*}=A^{*} A$ then $A$ is called a normal matrix; if $A=A^{*}$ then $A$ is called a Hermitian matrix; is $A A^{*}=I$ then $A$ is called a unitary matrix; for $B \in M_{n}(B \mathbb{C})$ if $A B=B A=I$ then $A$ is an invertible matrix.

Theorem 2.1. Let $A, B \in M_{n}(B \mathbb{C})$. Then the followings are satisfied.

$$
\begin{aligned}
& \text { i) }\left(A^{T}\right)^{-1}=\left(A^{-1}\right)^{T} \\
& \text { ii) a) }\left(\overline{A_{(i)}}\right)^{-1}=\overline{\left(A^{-1}\right)_{(i)}} \\
& \text { b) }\left(\overline{A_{(j)}}\right)^{-1}=\overline{\left(A^{-1}\right)_{(j)}} \\
& \text { c) }\left(\overline{A_{(i j)}}\right)^{-1}=\overline{\left(A^{-1}\right)_{(i j)}} \\
& \text { iii)a } \overline{A B_{(i)}}=\overline{A_{(i)} B_{(i)}} \\
& \text { b) } \overline{A B_{(j)}}=\overline{A_{(j)} B_{(j)}} \\
& \text { c) } \overline{A B_{(i j)}}=\overline{A_{(i j)} B_{(i j)}} \\
& \text { iv) }(A B)^{T}=B^{T} A^{T}
\end{aligned}
$$


Proof. Let $A=A_{1}+A_{2} j$ and $B=B_{1}+B_{2} j$, where $A_{1}, A_{2}, B_{1}$ and $B_{2}$ are complex matrices.Now we will prove the theorem.

i) We can easily show that the inverse of bicomplex matrix $A$ is

$$
A^{-1}=\frac{\operatorname{det} A_{1}}{\operatorname{det} A}\left(A_{1}\right)^{-1}+j \frac{\operatorname{det} A_{2}}{\operatorname{det} A}\left(A_{2}\right)^{-1} .
$$

So,using the properties of transpose and inverse we get

$$
A^{T}=\left(A_{1}+A_{2} j\right)^{T}=A_{1}^{T}+A_{2}^{T} j
$$

and

$$
\left(A^{T}\right)^{-1}=\left(A_{1}^{T}+A_{2}^{T} j\right)^{-1}=\frac{\operatorname{det} A_{1}^{T}}{\operatorname{det} A^{T}}\left(A_{1}^{T}\right)^{-1}+j \frac{\operatorname{det} A_{2}^{T}}{\operatorname{det} A^{T}}\left(A_{2}^{T}\right)^{-1} .
$$

On the other hand,we can write

$$
\begin{aligned}
& \left(A^{-1}\right)^{T}=\frac{\operatorname{det} A_{1}}{\operatorname{det} A}\left(A_{1}^{T}\right)^{-1}+j \frac{\operatorname{det} A_{2}}{\operatorname{det} A}\left(A_{2}^{T}\right)^{-1} \\
& \left(A^{-1}\right)^{T}=\frac{\operatorname{det} A_{1}^{T}}{\operatorname{det} A^{T}}\left(A_{1}^{T}\right)^{-1}+j \frac{\operatorname{det} A_{2}^{T}}{\operatorname{det} A^{T}}\left(A_{2}^{T}\right)^{-1}
\end{aligned}
$$

since $\operatorname{det} A=\operatorname{det} A^{T}$.Thus,we obtain

$$
\left(A^{-1}\right)^{T}=\left(A^{T}\right)^{-1}
$$

ii) a)According to the definition of bicomplex conguate of bicomplex number, we know

$$
\overline{A_{(i)}}=\overline{A_{1}}+\overline{A_{2}} j .
$$

After that we have

$$
\left(\overline{A_{(i)}}\right)^{-1}=\frac{\operatorname{det} \overline{A_{1}}}{\operatorname{det} \bar{A}_{(i)}}\left(\overline{A_{1}}\right)^{-1}+j \frac{\operatorname{det} \overline{A_{2}}}{\operatorname{det} \bar{A}_{(i)}}\left(\overline{A_{2}}\right)^{-1} .
$$

Furthermore,

$$
\begin{aligned}
{\overline{\left(A^{-1}\right)_{(i)}}} & =\overline{\left(\frac{\operatorname{det} A_{1}}{\operatorname{det} A}\left(A_{1}\right)^{-1}\right)}+j \overline{\left(\frac{\operatorname{det} A_{2}}{\operatorname{det} A}\left(A_{2}\right)^{-1}\right)} \\
{\overline{\left(A^{-1}\right)}}_{(i)} & =\frac{\overline{\operatorname{det} A_{1}}}{\overline{\operatorname{det} A_{(i)}}} \overline{\left(A_{1}\right)^{-1}}+j \frac{\overline{\operatorname{det} A_{2}}}{\overline{\operatorname{det} A_{(i)}}} \overline{\left(A_{2}\right)^{-1}} \\
{\overline{\left(A^{-1}\right)}}_{(i)} & =\frac{\operatorname{det} \overline{A_{1}}}{\operatorname{det} \bar{A}_{(i)}}\left(\overline{A_{1}}\right)^{-1}+j \frac{\operatorname{det} \overline{A_{2}}}{\operatorname{det} \bar{A}_{(i)}}\left(\overline{A_{2}}\right)^{-1} .
\end{aligned}
$$

So from the eq. (2.1) and (2.2) we find that

$$
\left(\overline{A_{(i)}}\right)^{-1}=\overline{\left(A^{-1}\right)_{(i)}}
$$

Conjugates of matrix $A$ respect to $j$ and $i j$ has also the same property.The proof of the case (b) and (c) are easily shown like the case (a).

iii) a) Since $A$ and $B$ are bicomplex matrices we have

$$
\begin{aligned}
A \cdot B & =A_{1} B_{1}-A_{2} B_{2}+\left(A_{1} B_{2}+B_{1} A_{2}\right) j \\
\overline{A \cdot B}{ }_{(i)} & =\overline{A_{1} B_{1}}-\overline{A_{2} B_{2}}+\left(\overline{A_{1} B_{2}}+\overline{B_{1} A_{2}}\right) j .
\end{aligned}
$$

From another direction,we can obtain

$$
\begin{aligned}
& \bar{A}_{(i)} \cdot \bar{B}_{(i)}=\overline{A_{1} B_{1}}+\overline{A_{1} B_{2}} j+\overline{B_{1} A_{2}} j-\overline{A_{2} B_{2}} \\
& \bar{A}_{(i)} \cdot \bar{B}_{(i)}=\overline{A_{1} B_{1}}-\overline{A_{2} B_{2}}+\left(\overline{A_{1} B_{2}}+\overline{B_{1} A_{2}}\right) j .
\end{aligned}
$$


Thus, we can write

$$
\overline{A B_{(i)}}=\overline{A_{(i)} B_{(i)}} .
$$

This property also applies to conjugates according to $j$ and $i j$.

iv) If we calculate $A B$ for $A$ and $B$ we have

$$
\begin{aligned}
& A . B=A_{1} B_{1}+A_{1} B_{2} j+B_{1} A_{2} j-A_{2} B_{2} \\
& A . B=A_{1} B_{1}-A_{2} B_{2}+\left(A_{1} B_{2}+A_{2} B_{1}\right) j .
\end{aligned}
$$

and using $A^{T}=A_{1}^{T}+A_{2}^{T} j$ and $B^{T}=B_{1}^{T}+B_{2}^{T} j$ we get

$$
(A \cdot B)^{T}=B_{1}^{T} A_{1}^{T}-B_{2}^{T} A_{2}^{T}+\left(B_{2}^{T} A_{1}^{T}+B_{1}^{T} A_{2}^{T}\right) j .
$$

On the other hand

$$
\begin{gathered}
B^{T} A^{T}=B_{1}^{T} A_{1}^{T}+B_{1}^{T} A_{2}^{T} j+B_{2}^{T} A_{1}^{T} j-B_{2}^{T} A_{2}^{T} \\
B^{T} A^{T}=B_{1}^{T} A_{1}^{T}-B_{2}^{T} A_{2}^{T}+\left(B_{2}^{T} A_{1}^{T}+B_{1}^{T} A_{2}^{T}\right) j .
\end{gathered}
$$

Thus, by using the eq. (2.3) and (2.4) we get

$$
(A B)^{T}=B^{T} A^{T} .
$$

Theorem 2.2. Let $A, B \in M_{n}(B \mathbb{C})$.Then the followings hold.

$$
\begin{aligned}
& \text { i)a) }\left(\overline{A_{(i)}}\right)^{T}={\overline{\left(A^{T}\right)}}_{(i)} \\
& \text { b) }\left({\overline{A_{(j)}}}^{T}={\overline{\left(A^{T}\right)}}_{(j)}\right. \\
& \text { c) }\left({\overline{A_{(i j)}}}^{T}={\overline{\left(A^{T}\right)}}_{(i j)}\right. \\
& \text { ii) a) }(A B)^{*}=B^{*} A^{*} \Rightarrow\left(\overline{A B}_{(i)}\right)^{T}=\left(\bar{B}_{(i)}\right)^{T}\left(\bar{A}_{(i)}\right)^{T} \\
& \text { b) }(A B)^{*}=B^{*} A^{*} \Rightarrow\left(\overline{A B}_{(j)}\right)^{T}=\left(\bar{B}_{(j)}\right)^{T}\left(\bar{A}_{(j)}\right)^{T} \\
& \text { c) }(A B)^{*}=B^{*} A^{*} \Rightarrow\left(\overline{A B}_{(i j)}\right)^{T}=\left(\bar{B}_{(i j)}\right)^{T}\left(\bar{A}_{(i j)}\right)^{T} \\
& \text { iii) }(A B)^{-1}=B^{-1} A^{-1} \text { if } A \text { and } B \text { are invertible. } \\
& \text { iv) a) }\left(A_{(i)}^{*}\right)^{-1}=\left(A^{-1}\right)_{(i)}^{*} \Rightarrow\left(\left(\overline{A_{(i)}}\right)^{T}\right)^{-1}=\left({\overline{\left(A^{-1}\right)}}_{(i)}\right)^{T} \\
& \text { b) }\left(A_{(j)}^{*}\right)^{-1}=\left(A^{-1}\right)_{(j)}^{*} \Rightarrow\left(\left(\bar{A}(j)^{T}\right)^{-1}=\left({\overline{\left(A^{-1}\right)}}_{(j)}\right)^{T}\right. \\
& \text { c) }\left(A_{(i j)}^{*}\right)^{-1}=\left(A^{-1}\right)_{(i j)}^{*} \Rightarrow\left(\left(\overline{A_{(i j)}}\right)^{T}\right)^{-1}=\left({\overline{\left(A^{-1}\right)}}_{(i j)}\right)^{T}
\end{aligned}
$$

Proof. As before, we write $A$ and $B$ as $A=A_{1}+A_{2} j, B=B_{1}+B_{2} j$, where $A_{1}, A_{2}, B_{1}$ and $B_{2}$ are $n \times n$ complex matrices.

i) a)It is easy to verify that

$$
\begin{gathered}
\overline{A_{(i)}}=\overline{A_{1}}+\overline{A_{2}} j \\
\left(\overline{A_{(i)}}\right)^{T}=\left(\overline{A_{1}}\right)^{T}-\left(\overline{A_{2}}\right)^{T} j \\
A^{T}=A_{1}^{T}+A_{2}^{T} j \\
{\overline{\left(A^{T}\right)_{(i)}}}=\overline{\left(A_{1}^{T}\right)}+\overline{\left(A_{2}^{T}\right)} j=\left(\overline{A_{1}}\right)^{T}-\left(\overline{A_{2}}\right)^{T} j .
\end{gathered}
$$

By using the eq. (2.5) and (2.6) we obtain

$$
\left(\overline{A(i)}^{T}={\overline{\left(A^{T}\right)}}_{(i)}\right.
$$

The same property also applies to conjugates according to $j$ and $i j$.The proof of the case (b) and (c) are easily shown like the case (a).

ii) a) For

$$
\begin{aligned}
& A^{*}=\left(\overline{A_{(i)}}\right)^{T}=\left(\overline{A_{1}+A_{2} j}\right)_{(i)}^{T}=\left(\overline{A_{1}}\right)^{T}+\left(\overline{A_{2}}\right)^{T} j \\
& B^{*}=\left(\overline{B_{(i)}}\right)^{T}=\left(\overline{B_{1}+B_{2} j}\right)_{(i)}^{T}=\left(\overline{B_{1}}\right)^{T}+\left(\overline{B_{2}}\right)^{T} j
\end{aligned}
$$


we calculate

$$
\begin{gathered}
B^{*} A^{*}=\left(\left(\overline{B_{1}}\right)^{T}+\left(\overline{B_{2}}\right)^{T} j\right)\left(\left(\overline{A_{1}}\right)^{T}+\left(\overline{A_{2}}\right)^{T} j\right) \\
B^{*} A^{*}=\left(\overline{B_{1}}\right)^{T}\left(\overline{A_{1}}\right)^{T}+\left(\overline{B_{1}}\right)^{T}\left(\overline{A_{2}}\right)^{T} j+\left(\overline{B_{2}}\right)^{T}\left(\overline{A_{1}}\right)^{T} j-\left(\overline{B_{2}}\right)^{T}\left(\overline{A_{2}}\right)^{T} .
\end{gathered}
$$

If we calculate $A B$ for $A$ and $B$ we have

$$
A B=\left(A_{1}+A_{2} j\right)\left(B_{1}+B_{2} j\right)=A_{1} B_{1}+A_{1} B_{2} j+A_{2} B_{1} j-A_{2} B_{2}
$$

then the conjugate transpose of $A B$

$$
\begin{gathered}
\left.(A B)^{*}=\overline{\left(A B_{(i)}\right)}\right)^{T}=\left(\overline{A_{1} B_{1}}+\overline{A_{1} B_{2}} j+\overline{A_{2} B_{1}} j-\overline{A_{2} B_{2}}\right)^{T} \\
(A B)^{*}=\left(\overline{B_{1}}\right)^{T}\left(\overline{A_{1}}\right)^{T}+\left(\overline{B_{1}}\right)^{T}\left(\overline{A_{2}}\right)^{T} j+\left(\overline{B_{2}}\right)^{T}\left(\overline{A_{1}}\right)^{T} j-\left(\overline{B_{2}}\right)^{T}\left(\overline{A_{2}}\right)^{T} .
\end{gathered}
$$

From using the eq. (2.5) and (2.6) the proof is completed.

Notice that the same property also applies to conjugates according to $j$ and $i j$.

iii) Since

$$
B^{-1} A^{-1} A B=B^{-1} I B=I=A B B^{-1} A^{-1}
$$

so

$$
(A B)^{-1}=B^{-1} A^{-1} .
$$

iv) a)Using the features of transpose and inverse we can obtain

$$
\left(\left(\bar{A}_{(i)}\right)^{T}\right)^{-1}=\frac{\operatorname{det}\left(\overline{A_{1}}\right)^{T}}{\operatorname{det}\left(\bar{A}_{(i)}\right)^{T}}\left(\left(\overline{A_{1}}\right)^{T}\right)^{-1}+j \frac{\operatorname{det}\left(\overline{A_{2}}\right)^{T}}{\operatorname{det}\left(\bar{A}_{(i)}\right)^{T}}\left(\left(\overline{A_{2}}\right)^{T}\right)^{-1} .
$$

On the other hand,

$$
\begin{aligned}
A^{-1} & =\frac{\operatorname{det} A_{1}}{\operatorname{det} A}\left(A_{1}\right)^{-1}+j \frac{\operatorname{det} A_{2}}{\operatorname{det} A}\left(A_{2}\right)^{-1} \\
{\overline{A^{-1}}}_{(i)} & =\frac{\overline{\operatorname{det} A_{1}}}{\operatorname{det} A_{(i)}}\left(A_{1}\right)^{-1}+j \frac{\overline{\operatorname{det} A_{2}}}{\operatorname{det} A_{(i)}}\left(A_{2}\right)^{-1} \\
{\overline{A^{-1}}}_{(i)} & =\frac{\overline{\operatorname{det} A_{1}}}{\overline{\operatorname{det} A_{(i)}}} \overline{\left(A_{1}\right)^{-1}}+j \frac{\overline{\operatorname{det} A_{2}}}{\overline{\operatorname{det} A_{(i)}}} \overline{\left(A_{2}\right)^{-1}} \\
{\overline{A^{-1}}}_{(i)} & =\frac{\operatorname{det} \overline{A_{1}}}{\operatorname{det} \overline{A_{(i)}}}\left(\overline{A_{1}}\right)^{-1}+j \frac{\operatorname{det} \overline{A_{2}}}{\operatorname{det} \overline{A_{(i)}}}\left(\overline{A_{2}}\right)^{-1} \\
\left({\overline{A^{-1}}}_{(i)}\right)^{T} & =\frac{\operatorname{det}\left(\overline{A_{1}}\right)^{T}}{\operatorname{det}\left(\bar{A}_{(i)}\right)^{T}}\left(\left(\overline{A_{1}}\right)^{-1}\right)^{T}+j \frac{\operatorname{det}\left(\overline{A_{2}}\right)^{T}}{\operatorname{det}\left(\bar{A}_{(i)}\right)^{T}}\left(\left(\overline{A_{2}}\right)^{-1}\right)^{T} \\
\left(\bar{A}_{(i)}\right)^{T} & =\frac{\operatorname{det}\left(\bar{A}_{1}\right)^{T}}{\operatorname{det}\left(\bar{A}_{(i)}\right)^{T}}\left(\left(\overline{A_{1}}\right)^{T}\right)^{-1}+j \frac{\operatorname{det}\left(\bar{A}_{2}\right)^{T}}{\operatorname{det}\left(\bar{A}_{(i)}\right)^{T}}\left(\left(\overline{A_{2}}\right)^{T}\right)^{-1} .
\end{aligned}
$$

Therefore, we get

$$
\left(A_{(i)}^{*}\right)^{-1}=\left(A^{-1}\right)_{(i)}^{*} \Rightarrow\left(\left({\overline{A_{(i)}}}^{T}\right)^{-1}=\left({\overline{\left(A^{-1}\right)}}_{(i)}\right)^{T}\right.
$$

by using eq.(2.9) and (2.10)

Conjugates of matrix $A$ respect to $j$ and $i j$ has also the same property.The proof of the case (b) and (c) are easily shown like the case (a).

Now we will define the complex adjoint matrix of a bicomplex matrix. After that we will give some relations between bicomplex matrices and their complex adjoint matrices. 
Definition 2.1. Let $A=A_{1}+A_{2} j \in M_{n}(B \mathbb{C})$ where $A_{1}, A_{2} \in M_{n}(\mathbb{C})$.The $2 n \times 2 n$ matrix

$$
\left(\begin{array}{cc}
A_{1} & A_{2} \\
-A_{2} & A_{1}
\end{array}\right)
$$

is called the bicomplex adjoint matrix of $\mathrm{A}$ and detoned $\chi_{A}$.

Example 2.1. Let $A=\left(\begin{array}{cc}1 & i \\ i j & i+j\end{array}\right)$ is a bicomplex matrix.

$$
\begin{aligned}
A & =A_{1}+A_{2} j \\
A & =\left(\begin{array}{ll}
1 & i \\
0 & i
\end{array}\right)+\left(\begin{array}{ll}
0 & 0 \\
i & 1
\end{array}\right) j
\end{aligned}
$$

The bicomplex adjoint matrix of $\mathrm{A}$ is

$$
\chi_{A}=\left(\begin{array}{cc}
A_{1} & A_{2} \\
-A_{2} & A_{1}
\end{array}\right)=\left(\begin{array}{cccc}
1 & i & 0 & 0 \\
0 & i & i & 1 \\
0 & 0 & 1 & i \\
-i & -1 & 0 & i
\end{array}\right) .
$$

Theorem 2.3. Let $A, B \in M_{n}(B \mathbb{C})$ Then the followings hold;

$$
\begin{aligned}
& \text { i) } \chi I_{n}=I_{2 n} \\
& \text { ii) } \chi_{A+B}=\chi_{A}+\chi_{B} \\
& \text { ii) } \chi_{A B}=\chi_{A} \chi_{B} \\
& \text { iv) } \chi_{A^{-1}}=\left(\chi_{A}\right)^{-1} \text { if } A^{-1} \text { exist; } \\
& \text { v) } \chi_{A^{*}} \neq\left(\chi_{A}\right)^{*} \text { in general } \\
& \text { vi) } \chi_{A} \text { is unitary, hermitian or normal if and only if } A \text { is unitary, hermitian or normal, respectively. }
\end{aligned}
$$

Proof. i)

$$
I_{n}=\left(\begin{array}{cccc}
1 & & & 0 \\
& \cdot & & \\
& & \cdot & \\
0 & & & 1
\end{array}\right)=I_{n}+0 j=A_{1}+A_{2} j
$$

Now we get the following equation;

$$
\chi I_{n}=\left(\begin{array}{cc}
A_{1} & A_{2} \\
-A_{2} & A_{1}
\end{array}\right)=\left(\begin{array}{cc}
I_{n} & 0 \\
0 & I_{n}
\end{array}\right)=I_{2 n} .
$$

ii) If we take $A=A_{1}+A_{2} j$ and $B=B_{1}+B_{2} j\left(A_{1}, A_{2}, B_{1} B_{2} \in M_{n}(\mathbb{C})\right)$

$$
\begin{aligned}
\chi_{A} & =\left(\begin{array}{cc}
A_{1} & A_{2} \\
-A_{2} & A_{1}
\end{array}\right) \text { and } \chi_{B}=\left(\begin{array}{cc}
B_{1} & B_{2} \\
-B_{2} & B_{1}
\end{array}\right) \\
A+B & =\left(A_{1}+B_{1}\right)+\left(A_{2}+B_{2}\right) j
\end{aligned}
$$

thus, we have;

$$
\chi_{A+B}=\left(\begin{array}{cc}
A_{1}+B_{1} & A_{2}+B_{2} \\
-A_{2}-B_{2} & A_{1}+B_{1}
\end{array}\right) .
$$

So for the complex adjoint matrix of $A+B$ we can write

$$
\chi_{A}+\chi_{B}=\left(\begin{array}{cc}
A_{1}+B_{1} & A_{2}+B_{2} \\
-A_{2}-B_{2} & A_{1}+B_{1}
\end{array}\right) .
$$

From the eq. (2.11) and (2.12) we obtain

$$
\chi_{A+B}=\chi_{A}+\chi_{B}
$$


iii)We know

$$
\begin{aligned}
& A B=\left(A_{1}+A_{2} j\right)\left(B_{1}+B_{2} j\right)=A_{1} B_{1}+A_{1} B_{2} j+A_{2} B_{1} j-A_{2} B_{2} \\
& A B=\left(A_{1} B_{1}-A_{2} B_{2}\right)+\left(A_{1} B_{2}+A_{2} B_{1}\right) j
\end{aligned}
$$

this equations are hold.Then we can find the complex adjoint matrix of $A B$

$$
\chi_{A B}=\left(\begin{array}{cc}
A_{1} B_{1}-A_{2} B_{2} & A_{1} B_{2}+A_{2} B_{1} \\
-A_{1} B_{2}-A_{2} B_{1} & A_{1} B_{1}-A_{2} B_{2}
\end{array}\right) .
$$

From the definition of complex adjoint of a bicomplex matrix and the product of matrices we get

$$
\chi_{A} \chi_{B}=\left(\begin{array}{cc}
A_{1} & A_{2} \\
-A_{2} & A_{1}
\end{array}\right)\left(\begin{array}{cc}
B_{1} & B_{2} \\
-B_{2} & B_{1}
\end{array}\right)=\left(\begin{array}{cc}
A_{1} B_{1}-A_{2} B_{2} & A_{1} B_{2}+A_{2} B_{1} \\
-A_{1} B_{2}-A_{2} B_{1} & A_{1} B_{1}-A_{2} B_{2}
\end{array}\right) .
$$

So the proof is completed by using the equations (2.13) and (2.14).

iv)Since $A A^{-1}=I$ we have

$$
\begin{aligned}
A A^{-1} & =I \Leftrightarrow \chi_{A} \chi_{A^{-1}}=I \\
& \Leftrightarrow \chi_{A} \chi_{A^{-1}}\left(\chi_{A^{-1}}\right)^{-1}=I\left(\chi_{A^{-1}}\right)^{-1} \\
& \Leftrightarrow \chi_{A} I=I\left(\chi_{A^{-1}}\right)^{-1} \\
& \Leftrightarrow \chi_{A}=\left(\chi_{A^{-1}}\right)^{-1} \\
& \Leftrightarrow\left(\chi_{A}\right)^{-1}=\chi_{A^{-1}} .
\end{aligned}
$$

v)If we calculate complex adjoint of $A$ with respect to $i$, then

$$
A_{(i)}^{*}=\left(\overline{A_{(i)}}\right)^{T}=\left(\overline{A_{1}}+\overline{A_{2}} j\right)^{T}=\left(\overline{A_{1}}\right)^{T}+\left(\overline{A_{2}}\right)^{T}
$$

and

$$
\chi_{A_{(i)}^{*}}=\left(\begin{array}{cc}
\left(\overline{A_{1}}\right)^{T} & \left(\overline{A_{2}}\right)^{T} \\
-\left(\overline{A_{2}}\right)^{T} & \left(\overline{A_{1}}\right)^{T}
\end{array}\right) .
$$

From another point of view,

$$
\begin{aligned}
\chi_{A} & =\left(\begin{array}{cc}
A_{1} & A_{2} \\
-A_{2} & A_{1}
\end{array}\right) \Rightarrow \overline{\chi_{A}}(i)=\left(\begin{array}{cc}
\overline{A_{1}} & \overline{A_{2}} \\
-\overline{A_{2}} & \overline{A_{1}}
\end{array}\right) \\
& \Rightarrow\left({\overline{\chi_{A}}}_{(i)}\right)^{T}=\left(\begin{array}{ll}
\overline{A_{1}} & \overline{-A_{2}} \\
\overline{A_{2}} & \overline{A_{1}}
\end{array}\right)
\end{aligned}
$$

and we get

$$
\left(\chi_{A}\right)_{(i)}^{*}=\left(\begin{array}{cc}
\overline{A_{1}} & \overline{-A_{2}} \\
\overline{A_{2}} & \overline{A_{1}}
\end{array}\right)
$$

From the eq. (2.15) and (2.16), we find this

$$
\chi_{A_{(i)}^{*}} \neq\left(\chi_{A}\right)_{(i)}^{*}
$$

vi)Let $\chi_{A}$ be a unitary matrix,then

$$
\begin{aligned}
\chi_{A}\left(\chi_{A}\right)_{(i)}^{*} & =I_{2 n}=\left(\begin{array}{ll}
I & 0 \\
0 & I
\end{array}\right) \\
& =\left(\begin{array}{cc}
A_{1} & A_{2} \\
-A_{2} & A_{1}
\end{array}\right)\left(\begin{array}{cc}
\left(\overline{A_{1}}\right)^{T} & \left(\overline{A_{2}}\right)^{T} \\
-\left(\overline{A_{2}}\right)^{T} & \left(\overline{A_{1}}\right)^{T}
\end{array}\right)
\end{aligned}
$$

and

$$
\chi_{A}\left(\chi_{A}\right)_{(i)}^{*}=\left(\begin{array}{cc}
A_{1}\left(\overline{A_{1}}\right)^{T}-A_{2}\left(\overline{A_{2}}\right)^{T} & A_{1}\left(\overline{A_{2}}\right)^{T}+A_{2}\left(\overline{A_{1}}\right)^{T} \\
-A_{2}\left(\overline{A_{1}}\right)^{T}-A_{1}\left(\overline{A_{2}}\right)^{T} & A_{2}\left(\overline{A_{2}}\right)^{T}-A_{1}\left(\overline{A_{1}}\right)^{T}
\end{array}\right)=\left(\begin{array}{ll}
I & 0 \\
0 & I
\end{array}\right) .
$$


From the eq (2.17) we have

$$
A_{1}\left(\overline{A_{1}}\right)^{T}-A_{2}\left(\overline{A_{2}}\right)^{T}=I \text { and } A_{1}\left(\overline{A_{2}}\right)^{T}+A_{2}\left(\overline{A_{1}}\right)^{T}=0 .
$$

On the other hand,

$$
\begin{aligned}
& A \cdot A_{(i)}^{*}=\left(A_{1}+A_{2} j\right)\left(\left(\overline{A_{1}}\right)^{T}+\left(\overline{A_{2}}\right)^{T} j\right) \\
& A \cdot A_{(i)}^{*}=A_{1}\left(\overline{A_{1}}\right)^{T}-A_{2}\left(\overline{A_{2}}\right)^{T}+\left(A_{1}\left(\overline{A_{2}}\right)^{T}+A_{2}\left(\overline{A_{1}}\right)^{T}\right) j .
\end{aligned}
$$

Now it is easy to verify that

$$
A \cdot A_{(i)}^{*}=I
$$

by using the eq. (2.17) and (2.18) .

Let $\chi_{A}$ be a hermitian matrix,then

$$
\begin{aligned}
\chi_{A} & =\left(\chi_{A}\right)_{(i)}^{*} \Leftrightarrow\left(\begin{array}{cc}
A_{1} & A_{2} \\
-A_{2} & A_{1}
\end{array}\right)=\left(\begin{array}{cc}
\left(\overline{A_{1}}\right)^{T} & \left(\overline{A_{2}}\right)^{T} \\
-\left(\overline{A_{2}}\right)^{T} & \left(\overline{A_{1}}\right)^{T}
\end{array}\right) \\
A_{1} & =\left(\overline{A_{1}}\right)^{T} \Rightarrow A_{1}=A_{1}^{*} \\
A_{2} & =\left(\overline{A_{2}}\right)^{T} \Rightarrow A_{2}=A_{2}^{*} \\
A & =A_{1}+A_{2} j \text { and } A_{(i)}^{*}=\left(\overline{A_{1}}\right)^{T}+\left(\overline{A_{2}}\right)^{T} j
\end{aligned}
$$

By using above equations we can get

$$
A=A_{(i)}^{*}
$$

Let $\chi_{A}$ be a normal matrix,then

$$
\begin{aligned}
\chi_{A} \cdot\left(\chi_{A}\right)_{(i)}^{*} & =\left(\chi_{A}\right)_{(i)}^{*} \cdot \chi_{A} \Leftrightarrow\left(\begin{array}{cc}
A_{1} & A_{2} \\
-A_{2} & A_{1}
\end{array}\right) \cdot\left(\begin{array}{cc}
\left(\overline{A_{1}}\right)^{T} & \left(\overline{A_{2}}\right)^{T} \\
-\left(\overline{A_{2}}\right)^{T} & \left(\overline{A_{1}}\right)^{T}
\end{array}\right) \\
& =\left(\begin{array}{cc}
\left(\overline{A_{1}}\right)^{T} & \left(\overline{A_{2}}\right)^{T} \\
-\left(\overline{A_{2}}\right)^{T} & \left(\overline{A_{1}}\right)^{T}
\end{array}\right) \cdot\left(\begin{array}{cc}
A_{1} & A_{2} \\
-A_{2} & A_{1}
\end{array}\right) \\
\left(A_{1}+A_{2} j\right)\left(\left(\overline{A_{1}}\right)^{T}+\left(\overline{A_{2}}\right)^{T} j\right) & =\left(\left(\overline{A_{1}}\right)^{T}+\left(\overline{A_{2}}\right)^{T} j\right)\left(A_{1}+A_{2} j\right) \\
& \Leftrightarrow A \cdot A_{(i)}^{*}=A_{(i)}^{*} \cdot A .
\end{aligned}
$$

Note that this proof is also satisfied for conjugate of matrix $A$ respect to $j$ and $i j$

Theorem 2.4. Let $A \in M_{n \times n}(B \mathbb{C})$. Then the followings are equivalent:

i) $A$ is invertible,

ii) $A X=0$ has a unique solution 0 ,

iii) $\left|\chi_{A}\right| \neq 0$, i.e., $\chi_{A}$ is an invertible,

iv) $A$ has no zero eigenvalue.More precisely if $A X=\lambda X$ or $A X=X \lambda$

for some bicomplex number $\lambda$ and some bicomplex vector

$X \neq 0$ then $\lambda \neq 0$.

Proof. $(i) \Rightarrow(i i)$ This part is trivial.

(ii) $\Rightarrow($ iii $)$ Let $A=A_{1}+A_{2} j, X=x_{1}+x_{2} j$ where $A_{1}, A_{2}$ are complex matrices and $x_{1}, x_{2}$ are complex column vectors.Then,

$$
\begin{aligned}
& A X=\left(A_{1}+A_{2} j\right)\left(x_{1}+x_{2} j\right) \\
& A X=\left(A_{1} x_{1}-A_{2} x_{2}\right)+\left(A_{1} x_{2}+A_{2} x_{1}\right) j
\end{aligned}
$$

From we can write,

$$
A_{1} x_{1}-A_{2} x_{2}=0 \text { and } A_{1} x_{2}+A_{2} x_{1}=0
$$


These equations give us the equations

$$
\begin{array}{r}
A_{1} x_{1}-A_{2} x_{2}=0 \\
-A_{1} x_{2}-A_{2} x_{1}=0
\end{array}
$$

So we get that

$$
A X=0 \text { if and only if }\left(\begin{array}{cc}
A_{1} & A_{2} \\
-A_{2} & A_{1}
\end{array}\right)\left(\begin{array}{c}
x_{1} \\
-x_{2}
\end{array}\right)=0
$$

That is $\chi_{A} \cdot\left(x_{1},-x_{2}\right)^{T}=0$ since $A X=0$ has an unique solution. $\chi_{A} \cdot\left(x_{1},-x_{2}\right)^{T}=0$ has an unique solution.Thus, since $\chi_{A}$ is a bicomplex matrix, $\chi_{A}$ is an invertible.

$(i i) \Rightarrow(i v)$ Let $A X=0$ has an unique solution 0 for $A \in M_{n \times n}(B \mathbb{C})$.Suppose that $\mathrm{A}$ has a zero eigenvalue.Then for some bicomplex vector $X \neq 0$ the equation $A X=\lambda X$ has a zero eigenvalue.Thus $A X=0$, and so by our assumption $X=0$, this is a contradiction. Now suppose that $\mathrm{A}$ has no zero eigenvalue.If we have $A X=0=\lambda X$ then by our assumption $X=0$.

$($ iii $) \Rightarrow(i)$ If $\chi_{A}$ is an invertible, then for $A=A_{1}+A_{2} j$ there exist a complex matrix $\left(\begin{array}{ll}B_{1} & B_{2} \\ B_{3} & B_{4}\end{array}\right)$ such that;

$$
\begin{aligned}
\left(\begin{array}{ll}
B_{1} & B_{2} \\
B_{3} & B_{4}
\end{array}\right)\left(\begin{array}{cc}
A_{1} & A_{2} \\
-A_{2} & A_{1}
\end{array}\right) & =\left(\begin{array}{ll}
I & 0 \\
0 & I
\end{array}\right) \\
B_{1} A_{1}-B_{2} A_{2} & =I \\
B_{1} A_{2}+B_{2} A_{1} & =0
\end{aligned}
$$

Using this equations, we can write

$$
\left(B_{1} A_{1}-B_{2} A_{2}\right)+\left(B_{1} A_{2}+B_{2} A_{1}\right) j=I
$$

That is $B A=I$ for $B_{1}=B_{1}+B_{2} j$.So A is an invertible bicomplex matrix.

\section{Eigenvalues of Bicomplex Matrix}

Definition 3.1. Let $A \in M_{n}(B \mathbb{C})$ and $\lambda \in B \mathbb{C}$.If $\lambda$ holds the equation $A x=\lambda x(A x=x \lambda)$

for some nonzero bicomplex column vector $x$, then $\lambda$ is called the left(right) eigenvalue of $A$.The set of the left eigenvalues $\delta_{l}(A)=\{\lambda \in B \mathbb{C}: A x=\lambda x$, for some $x \neq 0\}$ is called left spectrum of $A$.Similarly, the set of the right eigenvalues $\delta_{r}(A)=\{\lambda \in B \mathbb{C}: A x=x \lambda$, for some $x \neq 0\}$ is called right spectrum of A.

Theorem 3.1. If $A$ is a bicomplex $n \times n$ matrix, then the left and right eigenvalues of $A$ coincide; that is

$$
\delta_{l}(A)=\delta_{r}(A) .
$$

Proof. Let $\lambda$ be a left eigenvalue of A, i.e., $A x=\lambda x$ for some $x \neq 0$.For any bicomplex $q \neq 0$,we have;

$$
\begin{aligned}
\left(q A q^{-1}\right) q x & =\left(q \lambda q^{-1}\right) q x \\
A q x & =\left(q \lambda q^{-1}\right) q x
\end{aligned}
$$

since A is real.Taking $0 \neq q \in B \mathbb{C}$ such that $q A q^{-1}$ is a complex number and writing $q x=y=y_{1}+y_{2} j$, we have

$$
A y_{1}=y_{1} q \lambda q^{-1} \text { and } A y_{2}=y_{2} q \lambda q^{-1}
$$

It follows that $\lambda$ is a right eigenvalue of A.Similarly one can prove that every right eigenvalue is also a left eigenvalue.

Definition 3.2. Let $A \in M_{n}(B \mathbb{C})$ and $\chi_{A}$ be the complex adjoint matrix of $A$.We define the q-determinant of $A$ by $|A|_{q}=\left|\chi_{A}\right|$. Here $\left|\chi_{A}\right|$ is the usual determinant of $\chi_{A}$.It is immediate that,

i) $|A|_{q}=|A| \cdot\left|\bar{A}_{i}\right|$

ii) $|A|_{q}=|A| \cdot\left|\bar{A}_{j}\right|$

iii) $|A|_{q} \neq|A| \cdot\left|\bar{A}_{i j}\right|$ 
Example 3.1. Let $A=\left(\begin{array}{cc}1 & 0 \\ i j & i+j\end{array}\right)$ is a bicomplex matrix then $|A|=i+j$

$$
\begin{aligned}
& \overline{A_{i}}=\left(\begin{array}{cc}
1 & 0 \\
-i j & -i+j
\end{array}\right) \Rightarrow\left|\overline{A_{i}}\right|=-i+j \\
& |A|\left|\overline{A_{i}}\right|=(i+j)(-i+j)=-i^{2}+j^{2}=0 \\
& \overline{A_{j}}=\left(\begin{array}{cc}
1 & 0 \\
-i j & -i+j
\end{array}\right) \Rightarrow\left|\overline{A_{j}}\right|=i-j \\
& |A| \cdot\left|\overline{A_{j}}\right|=(i+j)(i-j)=i^{2}-j^{2}=0 \\
& \overline{A_{i j}}=\left(\begin{array}{cc}
1 & 0 \\
i j & -i-j
\end{array}\right) \Rightarrow\left|\overline{A_{i j}}\right|=-i-j \\
& |A| \cdot\left|\overline{A_{i j}}\right|=(i+j)(-i-j)=-i^{2}-i j-i j-j^{2}=2-2 i j \\
& A=A_{1}+A_{2} j=\left(\begin{array}{cc}
1 & 0 \\
0 & i
\end{array}\right)+\left(\begin{array}{cc}
0 & 0 \\
i & 1
\end{array}\right) j \\
& X_{A}=\left(\begin{array}{cccc}
1 & 0 & 0 & 0 \\
0 & i & i & 1 \\
0 & 0 & 1 & 0 \\
-i & -1 & 0 & i
\end{array}\right) \Rightarrow\left|X_{A}\right|=1 .\left|\begin{array}{ccc}
i & i & 1 \\
0 & 1 & 0 \\
-1 & 0 & i
\end{array}\right|=1 .\left|\begin{array}{cc}
i & 1 \\
-1 & i
\end{array}\right|=i^{2}+1=0 \\
& \left|X_{A}\right|=|A|_{q}=|A|\left|\overline{A_{i}}\right| \\
& \left|X_{A}\right|=|A|_{q}=|A|\left|\overline{A_{j}}\right| \\
& \left|X_{A}\right|=|A|_{q} \neq|A|\left|\overline{A_{i j}}\right| \text {. }
\end{aligned}
$$

\section{References}

[1] Y. Alagöz, K. H. Oral, and S. Yüce, "Split quaternion matrices", Miskolc Mathematical Notes vol.13(2012), No.2, p.223-232

[2] G.Baley Price, An İntroduction to multicomplex spaces and functions, 1991, Monographs and textbooks inpure and applied mathematics, v.140;Marcel Gekker, Inc., 402 pp

[3] F.O.Farid ,Qing-Wen Wang and F. Zhang, "On the eigenvalues of quaternion matrices", Linear and Multilinear Algebra vol.59, no.4, April 2011, 451-473

[4] H. Kaya, "Genelleştirilmiş Bikompleks sayılar" , Yüksek Lisans Tezi , Bilecik Şeyh Edebali Üniverstesi, Bilecik, 2014

[5] M.E.Luna-Elizarraras, M.Shapiro, D.C. Struppa, A.Vajiac, "Bicomplex Numbers and their elementary functions CUBO". A Mathematical Journal vol.14, No:02, (61-80). June, 2012

[6] M. Özdemir, M. Erdoğdu and H. Şimşek, "On the Eigen values and Eigenvectors of a Lorentzion Rotation Matrix by using split queternions". Adv.Appl. Clifford Algebras 24(2014), 179-192

[7] D.Rochon,M.Shapiro, "On algebraic properties of bicomplex and hyperbolic numbers", an. Univ. Oredea Fasc. Mat. 11 (2004) 71-110

[8] T. Ünal, "Kuaterniyonlar ve Kuaterniyon Matrisleri" , Yüksek Lisans Tezi, Dumlupınar Üniversitesi, Haziran, 2011.

[9] F. Zhang ,"Quaternions and Matrices of Quaternions" Linear Algebra Appl., vol 251, pp.21-57, 1997 


\section{Affiliations}

CANAN ÖLÇEK

AdDRESS: Usak University, Dept. of Mathematics, 64200, Usak-Turkey.

E-MAIL: cananolcek@gmail.com

ORCID ID:0000-0003-3079-3577

SEMRA KAYA NURKAN

ADDRESS: Usak University, Dept. of Mathematics, 64200, Usak-Turkey.

E-MAIL: semra.kaya@usak.edu.tr

ORCID ID:0000-0001-6473-4458 